\title{
Peculiarities of Synchronization in a Two-Layer Network of Chaotic Maps with Inhomogeneous Interlayer Coupling
}

\author{
T. R. Bogatenko, A. V. Bukh, G. I. Strelkova
}

This paper considers the effects of forced and mutual synchronization of complex spatiotemporal structures in a two-layer network of nonlocally coupled logistic maps in the presence of inhomogeneous interlayer coupling. Two different types of coupling topology are considered: the first one is the sparse interlayer coupling with randomly distributed coupling defects, and the second type is the cluster interlayer coupling, providing the coupling via designated finite groups of elements. The latter type of coupling topology is considered for the first time. As a quantitative measure of the synchronization effect on the network, variance averaged over time and variance averaged both over time and network elements are used. We analyze how the synchronization measure changes depending on a degree of the interlayer coupling sparseness. We also identify a cluster of network elements which can provide almost complete synchronization in the network under study when the interlayer coupling is introduced along them.

This paper is dedicated to the memory of our teacher and scientific supervisor Prof. Vadim S. Anishchenko who passed away last November.

Keywords: synchronization, sparse links, chimera state, multilayer network, interlayer coupling

Received February 26, 2021

Accepted March 22, 2021

This work was funded by the Deutsche Forschungsgemeinschaft (DFG, German Research Foundation) - Project number 163436311-SFB 910 and by RFBR and DFG according to the research project No. 20-52-12004.

Tatiana R. Bogatenko

trbogatenko@gmail.com

Andrei V. Bukh

buh.andrey@yandex.ru

Galina I. Strelkova

strelkovagi@sgu.ru

Saratov State University

ul. Astrakhanskaya 83, Saratov, 410012 Russia

RUSSIAN JOURNAL OF NONLINEAR DYNAMICS, 2021, 17(1), 103-117 


\section{Introduction}

Synchronization is one of important and fundamental phenomena of self-organization of nonlinear self-oscillating systems [1-6]. Recently, there has been great interest in the study of the dynamics of interconnected complex ensembles and networks and, in particular, various forms of synchronization: cluster [7, 8], generalized [9], relay [10-14], forced and mutual [15-22], intralayer and interlayer [23-26], and explosive synchronization [27-32].

In real life, there are often cases of breaking or absence of connections between interacting ensembles, due to the influence of various external factors or the peculiarities of the functioning of the systems under consideration. The analysis of inhomogeneity of couplings is of great practical importance in the transmission of information [33], ensuring the stable functioning of energy networks [34-36], as well as in neurodynamics and neurophysiology [37-39]. Thus, it seems sensible and urgent to study the influence of the absence of connecting elements on the synchronization of interacting ensembles. The role of communication nodes on the ability of a network of coupled systems to synchronize was considered in $[23,25,40]$. The problem of sparse coupling was particularly raised and described in [24,41] for multiplex networks of continuous-time systems. However, the authors did not consider how the sparse coupling can affect synchronization of complex spatio-temporal structures, such as chimera states. In addition, the forced synchronization effect was not studied either. The first attempt to analyze the influence of the interlayer coupling sparseness on the dynamics and synchronization of complex patterns was made in [42] for the case of an inhomogeneous multiplex network of chaotic maps. However, in this work only the case of regularly de-multiplexed nodes was investigated. It is also worth noting that synchronization effects in multilayer networks have not yet been explored for the case where the interlayer coupling is introduced through specially or arbitrary chosen groups of elements.

In the present paper we continue to study the influence of the inhomogeneous interlayer coupling on the synchronization of complex multiplex networks. We investigate effects of forced and mutual synchronization of chimera states in a two-layer network of discrete-time systems for two configurations of the interlayer coupling: (i) the sparse coupling with randomly distributed de-multiplexed elements and (ii) the "cluster" coupling when the layers interact only via certain finite groups of elements. The cluster interlayer coupling topology is considered for the first time. Each layer in the network under study represents a ring of nonlocally coupled logistic maps, which can exhibit amplitude and phase chimeras $[43,44]$. We analyze the degree of synchrony of chimera states when the number of removed links changes. We also study and identify a cluster of the coupled nodes, which provides almost complete synchronization of chimera states in the network considered.

\section{The model under study and research methods}

In our numerical simulation we deal with a two-layer network of nonlocally coupled chaotic oscillators which is described by the following equations:

$$
\begin{aligned}
& x_{i}^{t+1}=f_{i}^{t}\left(x_{i}^{t}\right)+\frac{\sigma_{1}}{2 P} \sum_{j=i-P}^{i+P}\left[f_{j}^{t}\left(x_{j}^{t}\right)-f_{i}^{t}\left(x_{i}^{t}\right)\right]+\gamma_{21}(i) F_{i}^{t}, \\
& y_{i}^{t+1}=g_{i}^{t}\left(y_{i}^{t}\right)+\frac{\sigma_{2}}{2 R} \sum_{j=i-R}^{i+R}\left[g_{j}^{t}\left(y_{j}^{t}\right)-g_{i}^{t}\left(y_{i}^{t}\right)\right]+\gamma_{12}(i) G_{i}^{t} .
\end{aligned}
$$


In (2.1) $x$ and $y$ are dynamical variables, $i$ is the number of an element, $i=1, \ldots, N=1000$ is the total number of oscillators in each layer, $t$ denotes discrete time. The behavior of individual nodes in (2.1) is described by logistic maps $f_{i}^{t}\left(x_{i}^{t}\right)=\alpha_{1} x_{i}^{t}\left(1-x_{i}^{t}\right), g_{i}^{t}\left(y_{i}^{t}\right)=\alpha_{2} y_{i}^{t}\left(1-y_{i}^{t}\right)$ with bifurcation parameters $\alpha_{1}$ and $\alpha_{2}$. In this paper, we consider $\alpha_{1}=\alpha_{2}=3.8$, which corresponds to chaotic dynamics of each map. $P$ and $R$ are the number of neighbors to the left and right from the $i$ th oscillator in each layer. These parameters specify the ranges of nonlocal intralayer coupling. $\sigma_{1}$ and $\sigma_{2}$ define nonlocal coupling strengths between the elements in each layer.

The coupling between the layers is organized as a multiplex, i.e., the $i$ th element of the first layer is coupled with the $i$ th element in the second layer, and is defined by the coupling functions $F_{i}^{t}$ and $G_{i}^{t}$ which determine the dissipative interaction: $F_{i}^{t}=\left(g_{i}^{t}\left(y_{i}^{t}\right)-f_{i}^{t}\left(x_{i}^{t}\right)\right)$, $G_{i}^{t}=\left(f_{i}^{t}\left(x_{i}^{t}\right)-g_{i}^{t}\left(y_{i}^{t}\right)\right)$. Coefficients $\gamma_{21}(i)$ and $\gamma_{12}(i)$ represent the coupling strengths between the layers, or interlayer coupling strengths. In the present research, these coefficients are functions of the elements' numbers which provide the interlayer coupling. The elements themselves are chosen randomly following a uniform distribution, i.e., there is a random sequence of connection nodes for which $\gamma_{21}(i)$ and $\gamma_{12}(i)$ are not equal to zero. For the rest of the oscillators in the system the interlayer coupling coefficients are equal to zero.

When studying forced synchronization, the first $(x)$ layer is considered to be a driving ring (driver), and the second $(y)$ layer, a driven ring (response). In this case, the interlayer coupling is unidirectional and $\gamma_{21}(i)=0, \gamma_{12}(i)=\gamma(i)>0$ for the chosen elements. When considering mutual synchronization, both layers impact each other, so the interlayer coupling becomes bidirectional and $\gamma_{21}(i)=\gamma_{12}(i)=\gamma(i)>0$ for the chosen elements.

Equations (2.1) are solved numerically for periodic boundary conditions (the layers are considered to be rings) and for fixed parameters of the intralayer coupling $\sigma_{1}=\sigma_{2}=0.32$ and $P=R=320$, at which phase and amplitude chimera states can be observed in an isolated ring $[43,44]$. The initial conditions are randomly distributed across elements $i=1,2, \ldots, N$ on the interval $[0 ; 1]$. This enables us to observe different chimera structures in the identical layers. A time interval of $3.5 \times 10^{5}$ is allotted for the system to settle and demonstrate steady regimes; iteration time itself is $10^{6}$. In order to illustrate spatio-temporal dynamics of the system, spatiotemporal profiles of the layers for $x_{i}$ and $y_{i}$ variables are constructed [44]. In this case, the first 30 iterations after the transient period are plotted in the graph for each element $i=1,2, \ldots, N$.

Figure 1 exemplifies spatio-temporal structures in the first $(x)$ (Fig. 1a) and the second $(y)$ (Fig. 1b) layers when there is no interlayer coupling between them. In the first layer one can detect a spatio-temporal regime which corresponds to the coexistence of incoherent clusters

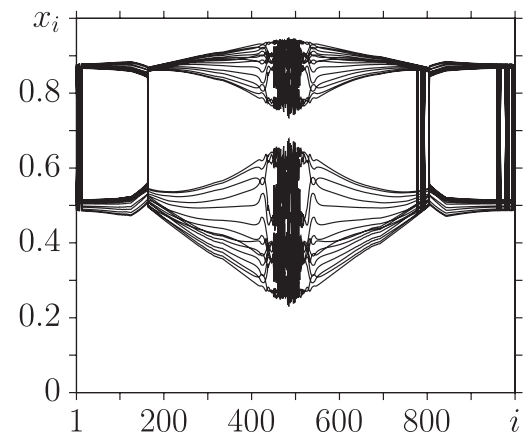

(a)

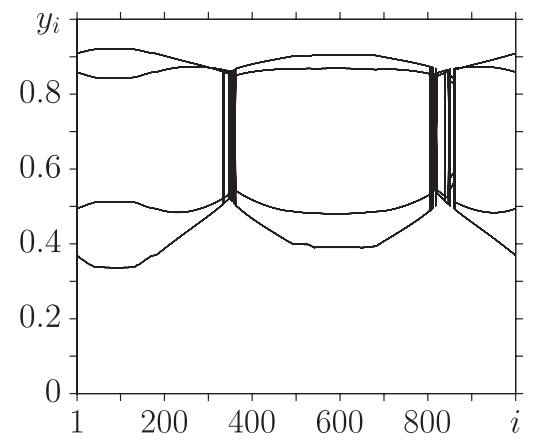

(b)

Fig. 1. Spatio-temporal profiles of the first (a) and the second (b) layers without interlayer coupling. The parameters are: $\alpha_{1}=\alpha_{2}=3.8, \sigma_{1}=\sigma_{2}=0.32$, and $P=R=320$. 
of amplitude (450 $\leqslant i \leqslant 550$ oscillators) and phase (clusters $0 \leqslant i \leqslant 10,160 \leqslant i \leqslant 170$, $780 \leqslant i \leqslant 800$ and $980 \leqslant i \leqslant 1000$ of oscillators) chimera states (Fig. 1a). The second layer exhibits the regime of phase chimera states (incoherent clusters $360 \leqslant i \leqslant 380$ and $800 \leqslant i \leqslant 830$ of oscillators) (Fig. 1b). The structures denoted are considered initial for all the undermentioned numerical experiments.

As there are complex spatio-temporal structures (Fig. 1) in the ensembles, it is sensible to estimate the synchronization effects in coherent and noncoherent clusters of chimera structures in the system. In order to do it, we calculate the root-mean-square variances between the symmetric elements of the rings. The first characteristic to consider is variance averaged over time, which is used to determine the clusters that respond to the impact and synchronize faster as the interlayer coupling strength grows. This synchronization measure is defined as follows:

$$
\delta_{i}=\sqrt{\frac{1}{n} \sum_{k=1}^{n}\left(y_{i}(k)-x_{i}(k)\right)^{2}} .
$$

In (2.2) $n$ is averaging time, equal to the iteration time. This quantity is computed for each element $i=1,2, \ldots, N$ of $(2.1)$ as the intercoupling strength changes.

To get a general (global) estimation of the synchronization effects in the network (2.1), we use variance averaged both over time and network elements. The second synchronization measure is calculated as follows:

$$
\Delta=\sqrt{\frac{1}{N} \sum_{i=1}^{N} \sqrt{\frac{1}{n} \sum_{k=1}^{n}\left(y_{i}(k)-x_{i}(k)\right)^{2}}}
$$

In (2.3) $N$ is the number of oscillators in each layer, and $n$ is averaging time.

In the case of identical interacting layers, complete synchronization of spatio-temporal structures takes place if $\delta_{i}=0$ for all $i=1,2, \ldots, N$ and $\Delta=0$. If these equalities are true only for a finite group of network (2.1) elements, then cluster synchronization of the structure takes place. If there is some heterogeneity in the studied network (in terms of the control parameter, and/or intralayer coupling parameters), the synchronization measures (2.2) and (2.3) will no longer be exactly zero. In this case, imposing some smallness conditions for the quantities $\delta_{i}$ and $\Delta$, we observe effective synchronization of the structures of interacting ensembles.

Figure 2 shows dependences of the measure $\Delta(2.3)$ on interlayer coupling strength $\gamma$ for unidirectional (Fig. 2a) and bidirectional (Fig. 2b) interlayer coupling without any interlayer coupling defects in the network (2.1) [20]. It is seen that in both cases, starting with a certain value of $\gamma, \Delta=0$. This means that the structures in the layers become completely identical and thus, complete synchronization of spatio-temporal patterns occurs in the network (2.1). Note that as follows from Fig. 2b, mutual synchronization is observed within a finite interval of $\gamma$, that is typical for this form of synchronization [20,21].

\section{Synchronization phenomenon with sparse interlayer coupling}

Let us examine the influence of interlayer coupling sparseness on the forced and mutual synchronization phenomena in the network (2.1) when the interlayer coupling strength is varied. In the experiments conducted the couplings between the layers were removed in a random uniform distribution, as was mentioned earlier. In this paper, we will depict the results of numerical 


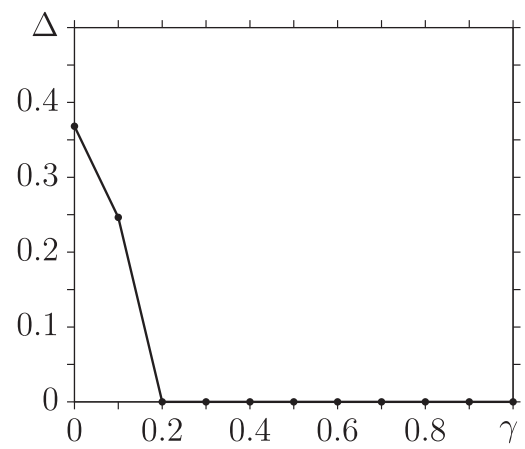

(a)

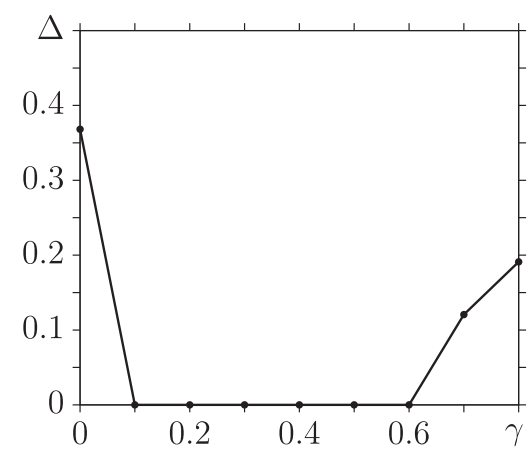

(b)

Fig. 2. Dependences of $\Delta$ (2.3) on interlayer coupling strength $\gamma$ for unidirectional (a) and bidirectional (b) interlayer coupling in the network (2.1) in the full-multiplex case. The parameters are: $\alpha_{1}=$ $=\alpha_{2}=3.8, \sigma_{1}=\sigma_{2}=0.32$ and $P=R=320$.

analysis of the synchronization effects in the system in the case of randomly distributed interlayer coupling defects. Let us detail the features of forced and mutual synchronization of chimera states, when $25 \%$ interlayer couplings are randomly removed in the network (2.1).

1. Unidirectional coupling with $\mathbf{2 5 \%}$ defects. Figures 3 and 4 a show spatio-temporal profiles of the structures in the second layer and the results of calculation of the 2nd synchronization measure $\Delta$ as the interlayer coupling strength increases.

As can be seen in the plots, in the second (response) ring effective synchronization of the chimera structure takes place under the impact of the driver (Fig. 1a). For all the $\gamma$ values $\Delta$ is not equal to zero and is not larger than 0.12 (Fig. 4a). The best coincidence of the structures of the driver and the response layers is observed at weak coupling strength $\gamma=0.1$ (Fig. 3a), at which $\Delta$ value reaches its minimum $(\Delta \approx 0.08)$. With further increase of the unidirectional coupling $\gamma>0.1$, the response ring qualitatively reproduces the structure of the driver (Figs. 3b, 3c), but the difference in the amplitude values of the oscillators in the coupled rings becomes quite noticeable.

2. Bidirectional coupling at $\mathbf{2 5 \%}$ defects. As the numerical analysis has shown, the lack of $25 \%$ of interlayer connections does not have a strong impact on the establishment of

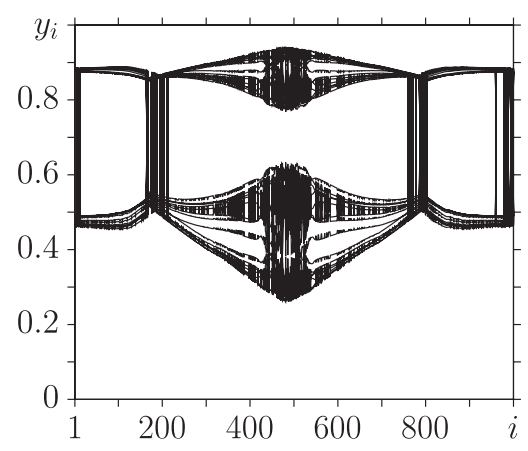

(a)

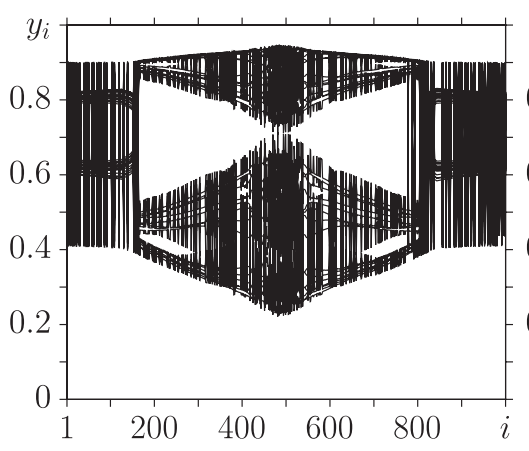

(b)

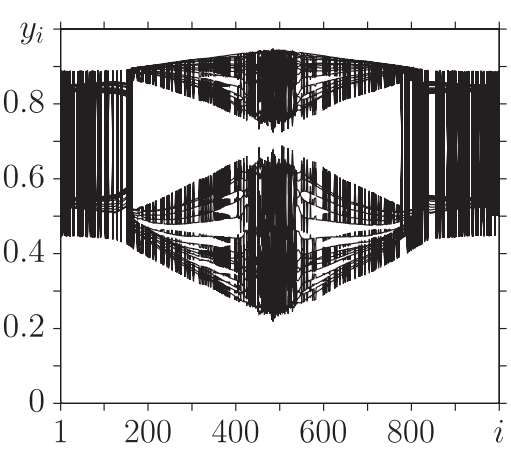

(c)

Fig. 3. Spatio-temporal profiles of the structures in the second (response) ring for various values of unidirectional intercoupling strength $\gamma$ : (a) 0.1 , (b) 0.2 , (c) 0.6 , in the absence of $25 \%$ of interlayer connections. The other parameters are as for Fig. 1. 
mutual synchronization between the layers (2.1). The two rings of the logistic maps influence one another, which results in the synchronization of spatio-temporal structures different from the initial ones (Fig. 1). As is illustrated in Fig. 5, both chimera states (Figs. 5a, 5c) and almost spatially homogeneous (Fig. 5b) structures can be synchronized. Besides, the differences in spatio-temporal dynamics of the interacting rings are quite insignificant. Figure $4 \mathrm{~b}$ shows that $\Delta<0.08$ on the interval of $\gamma \in[0.2,0.6]$, which corresponds to the effective mutual synchronization phenomenon, while the minimum $\Delta \approx 0.02$ occurs at $\gamma=0.3$ (Fig. $4 \mathrm{~b}$ ).

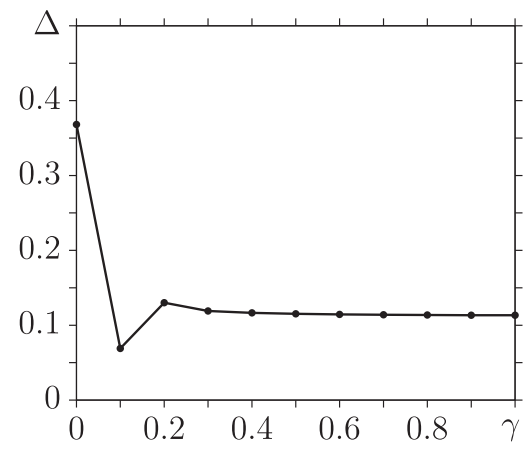

(a)

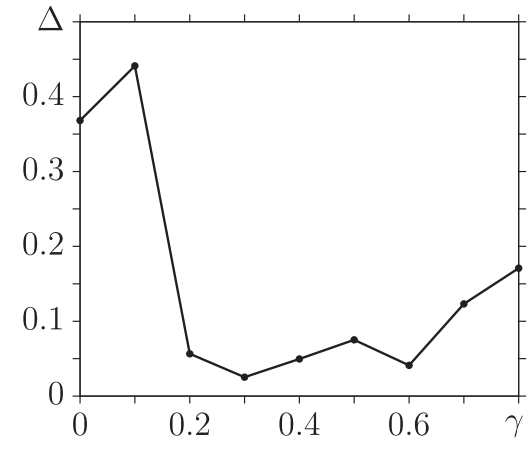

(b)

Fig. 4. Synchronizaton measure (2.3) as a function of interlayer coupling strength $\gamma$ for unidirectional (a) and bidirectional (b) interlayer coupling in the network (2.1) in the absence of $25 \%$ of interlayer connections removed randomly. The other parameters are as for Fig. 1.
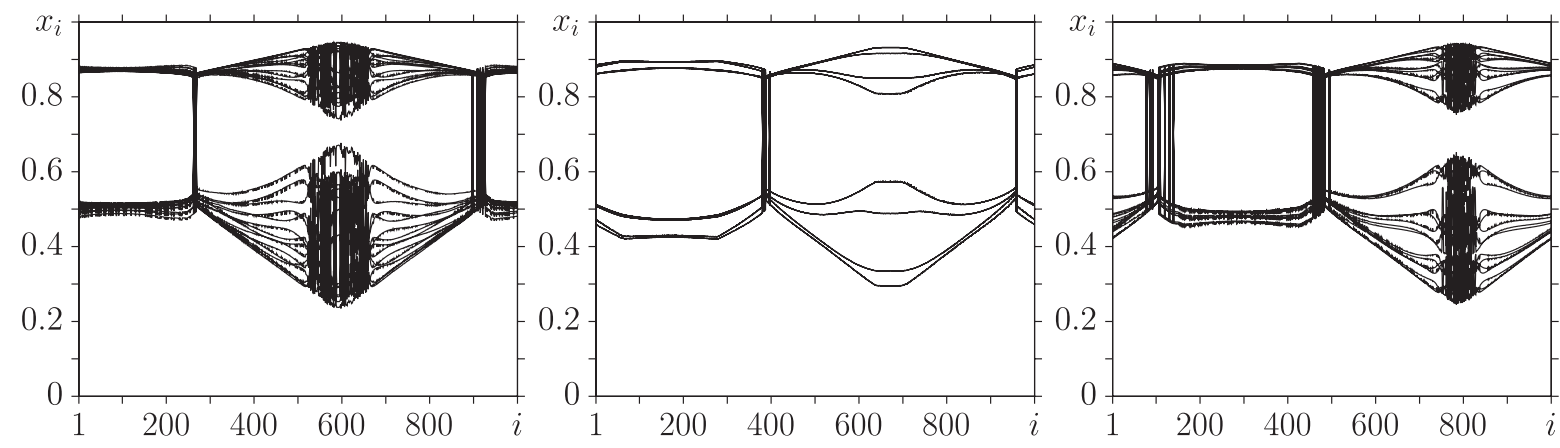

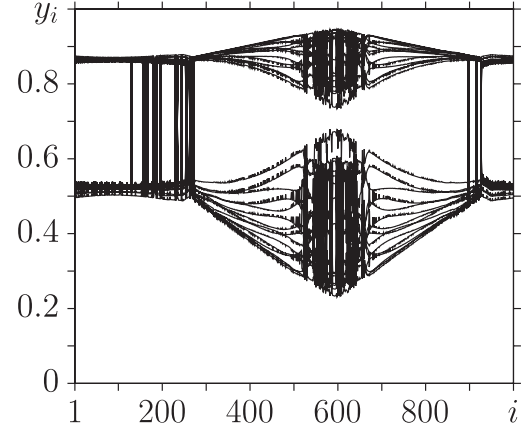

(a)

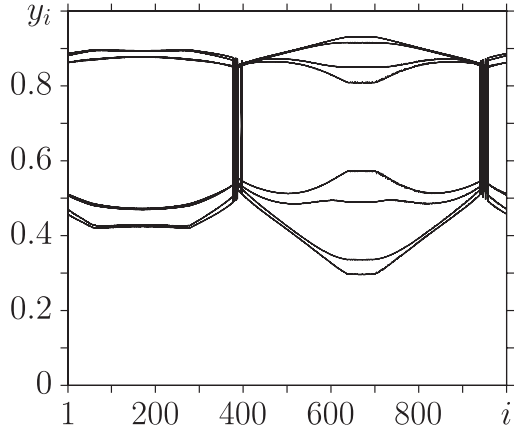

(b)

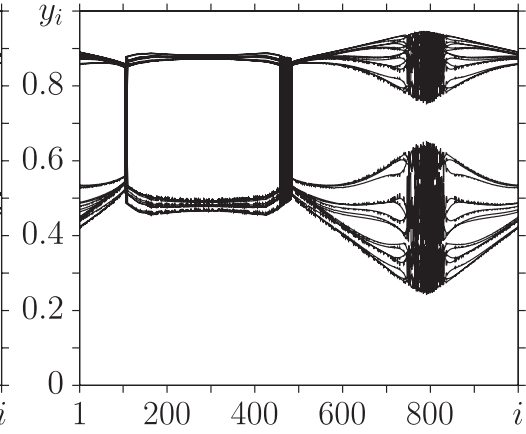

(c)

Fig. 5. Spatio-temporal profiles of the structures in the first (the upper row) and the second (the lower row) layers for various values of bidirectional interlayer coupling strength $\gamma$ : (a) 0.2 , (b) 0.3 , (c) 0.6 , in the absence of $25 \%$ of interlayer connections. The other parameters are as for Fig. 1. 
3. Analysis of the influence of the interlayer coupling sparseness. The calculations carried out for a different number of interlayer coupling defects showed that, as the number of coupled oscillators decreases, the average minimum value of $\Delta(2.3)$ changes. To obtain a more detailed picture of the impact of the number of randomly assigned intercoupling defects, the arithmetic mean values of $\Delta$ were calculated for all the values of the interlayer coupling strength for a different number of removed links. The corresponding dependences are shown in Fig. 6 for unidirectional (Fig. 6a) and bidirectional (Fig. 6b) coupling between the layers (2.1).

From the plots one can notice the following. For unidirectional coupling, as the number of coupled oscillators decreases, the measure $\Delta$ monotonically increases (Fig. 6a); this effect is selfexplanatory and the reasons for it are obvious. When the interlayer coupling becomes sparser, the response ring repeats the structure of the driver less and less accurately. As a result, the value of $\Delta$ increases with an increase in the number of de-multiplexed links. But for bidirectional interlayer coupling the $\Delta(\gamma)$ graph resembles a resonance curve and is characterized by a maximum at $50 \%$ of intercoupling defects (Fig. $6 \mathrm{~b}$ ). For more than $50 \%$ of defects the synchronization measure $\Delta(2.3)$ start to decrease.

The confirmation of these processes can be found in the two-parameter diagrams in the "interlayer coupling strength - percent of missing links" parameter plane, as is shown in Fig. 7. In these figures, the regions of synchronization and desynchronization can be distinguished in the case of unidirectional (Fig. 7a) and mutual (Fig. 7b) interlayer coupling in the system (2.1). As a quantitative assessment of the synchronization degree, the values of $\Delta(2.3)$ were used.

As the diagrams show, with increasing number of interlayer coupling defects, the time and ensemble variance grows, which is indicative of deterioration or even lack of effective external synchronization. If we take the fulfillment of the $\Delta<0.15$ condition as a criterion of synchronization, we can identify regions with effective synchronization from the interlayer coupling strength $\gamma$ and the number of missing interlayer links. As can be seen from the plot of Fig. 7a, the effective synchronization (in the sense of the fulfillment of the aforementioned condition) is achieved when $\gamma>0.2$ and when the number of the missing intercoupling links is no more than $38 \%$. In addition, for weak interlayer coupling, $0.03<\gamma<0.18$ (Fig. 7a), effective synchronization can also be achieved for a larger number of defects (up to $70 \%$ ).

For bidirectional coupling between the layers (Fig. 7b) one can also observe a finite interval of the interlayer coupling strength $0.18<\gamma<0.6$, within which effective mutual synchronization is achieved for $1-38 \%$ of missing links.

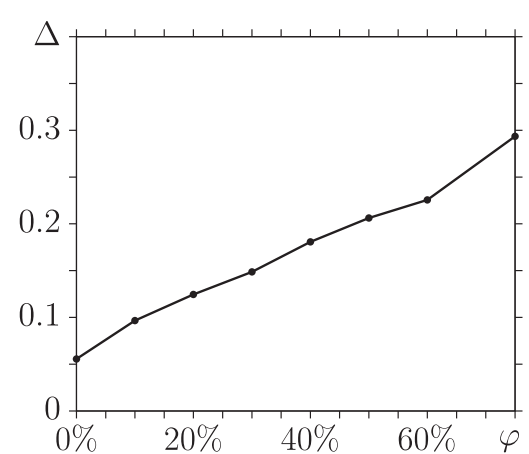

(a)

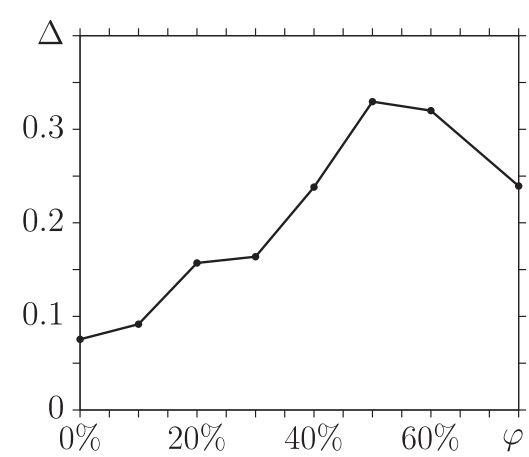

(b)

Fig. 6. Dependences of arithmetic mean values of synchronization measure $\Delta(2.3)$ on the percentage of the removed links for unidirectional (a) and bidirectional (b) interlayer coupling. The other parameters are as for Fig. 1. 


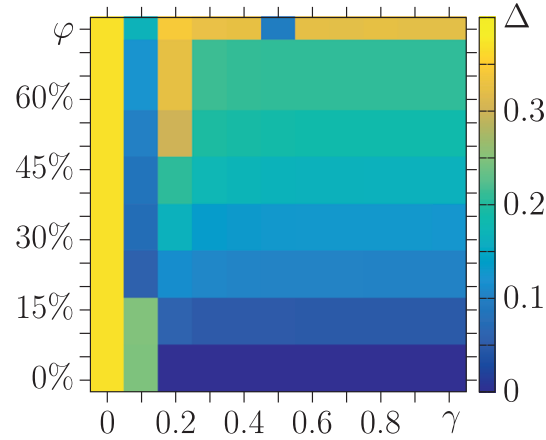

(a)

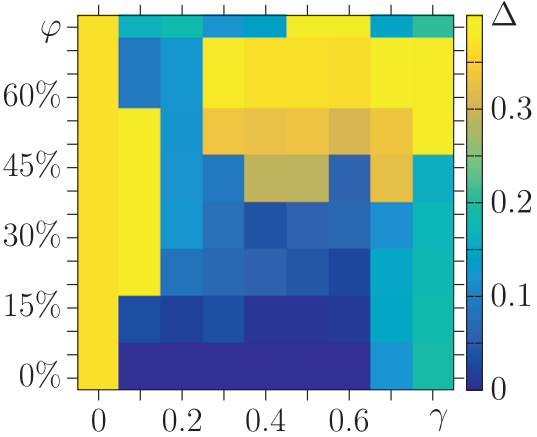

(b)

Fig. 7. Two-parameter diagrams of $\Delta$ in the $(\gamma, \%$ of missing links) parameter plane for unidirectional (a) and bidirectional (b) interlayer coupling. The other parameters are as for Fig. 1.

Nevertheless, for the general tendency of synchronization measure $\Delta$ growth with increasing number of defects, the diagrams show some anomalies in the form of areas of low values of $\Delta$ with significant defects. The reasons for these phenomena are still to be explored.

\section{The impact of cluster interlayer coupling on the synchronization effects}

Let us continue with the numerical analysis of the dynamics of the two-layer network (2.1) in the case of the so-called cluster interlayer coupling, when we remove all the coupling links between the layers except for those that belong to the desired cluster or a group of oscillators in the first $(x)$ ring. Using the initial state of the first layer (Fig. 1a), one can distinguish four different clusters of elements, which can be used to introduce cluster intercoupling: (1) via a noncoherent cluster of an amplitude chimera (oscillators in $[450 ; 520]$ ); (2) via a noncoherent cluster of a phase chimera (oscillators in [0;10], [160; 170], [750; 810] and [960; 999]); (3) via coherent regions (oscillators in $[10 ; 160]$ and $[810 ; 960]$ ) and (4) via noncoherent regions in the vicinity of an amplitude chimera state (oscillators in [170; 450] and [520; 750]). In these cases, the intercoupling strength coefficients in (1) are nonzero only for the elements that belong to the aforementioned clusters. As examples, we present the results of calculations for cases (1) and (4) of the cluster interlayer coupling.

1. Coupling via a noncoherent cluster of an amplitude chimera. To begin with, let us consider the case when the layers are uni- and bidirectionally coupled via a noncoherent cluster of an amplitude chimera in the interval from the 450 th to the 520 th oscillator (Fig. 1a). The results of calculations of spatio-temporal structures which can be observed in the response $(y)$ layer with the increase in coupling strength are presented in Fig. 8. The corresponding changes in the synchronization measure (2.3) value are shown in Fig. 9a, in the form of the graph $\Delta(\gamma)$.

As can be noticed from the spatio-temporal profiles (Fig. 8), having unidirectional interlayer coupling in the network (2.1), one can achieve structures which resemble the initial state of the driver $((x))$ (Fig. 1a). Also, as can be seen from the graph in Fig. 9a, the value of $\Delta$ is quite large even at low values of the interlayer coupling strength, for example, $\Delta>0.15$. As the strength of the interlayer coupling increases $(\gamma \geqslant 0.7)$, the synchronization measure becomes $\Delta \approx 0.3$. Thus, in this case, one cannot speak of effective forced synchronization of the structures. 


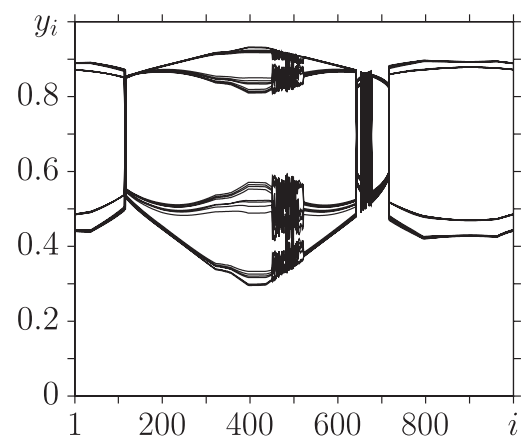

(a)

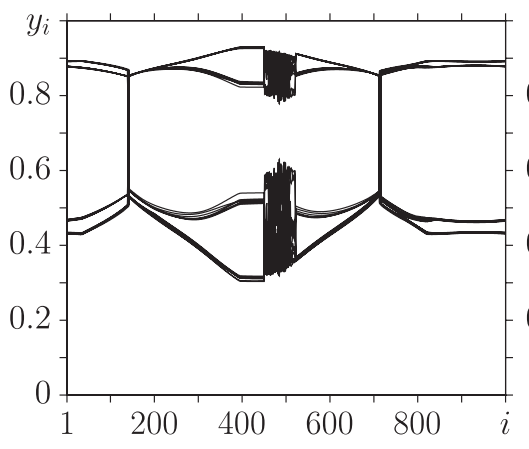

(b)

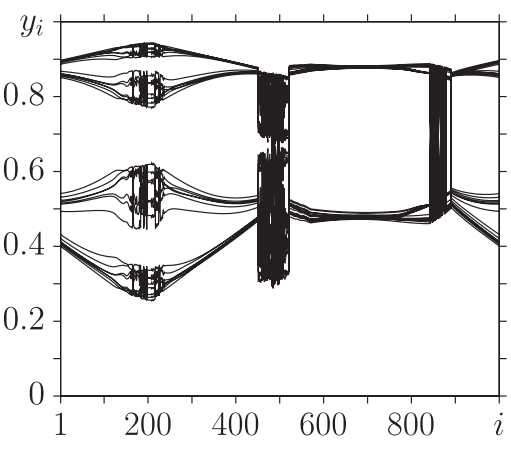

(c)

Fig. 8. Spatio-temporal profiles of the structures in the second $y$ layer for various values of intercoupling strength $\gamma$ : (a) 0.1 , (b) 0.4, (c) 0.9, with the unidirectional coupling via a noncoherent cluster of an amplitude chimera state. The other parameters are as for Fig. 1.

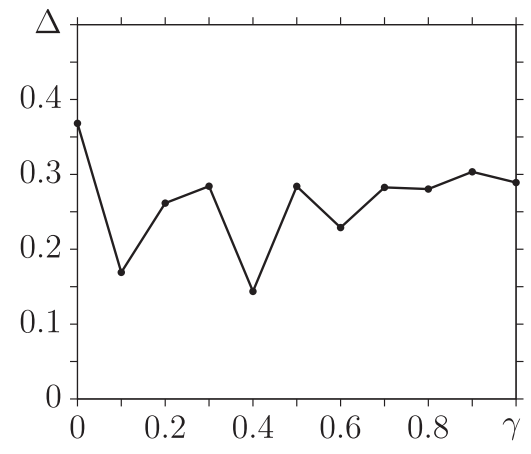

(a)

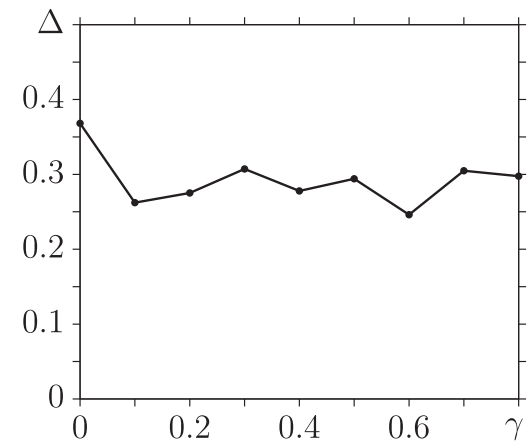

(b)

Fig. 9. Dependences of $\Delta$ (2.3) on interlayer coupling strength $\gamma$ for unidirectional (a) and bidirectional (b) interlayer coupling in the network (2.1) with the coupling via a noncoherent cluster of an amplitude chimera state. The other parameters are as for Fig. 1.

In addition, in the spatio-temporal profiles (Fig. 8) one can notice the existence of an oscillator region $i \in[450 ; 520]$ whose dynamics is drastically different from the rest of the response ring. This group of elements corresponds to the amplitude chimera cluster, through which the layers are coupled. The localization of this cluster does not change with an increase in the intercoupling strength, and all of its elements exhibit developed chaotic dynamics. At the same time, other regions in the space of the response layer undergo significant changes when $\gamma$ changes, which leads to rather large values of $\Delta$ (Fig. 9a).

In the case of mutual interlayer coupling between the layers through an noncoherent cluster of the amplitude chimera of the first layer, a qualitatively similar picture is observed. The established spatio-temporal structures in interacting layers noticeably differ from each other when the coupling strength $\gamma$ is being varied. The differences are clearly visible from the spatiotemporal profiles of the structures shown in Fig. 10, and reflected in the dependence $\Delta(\gamma)$ (Fig. 9b). For all values of $\gamma>0.1, \Delta>0.25$. Hence, in this case, we cannot observe effective mutual synchronization of structures.

It can be seen from Fig. 10 that, as in the case of unidirectional coupling, the localization of the region in the space of layers corresponding to the cluster of interlayer coupling (oscillators $i \in[450 ; 520])$ remains unchanged. 

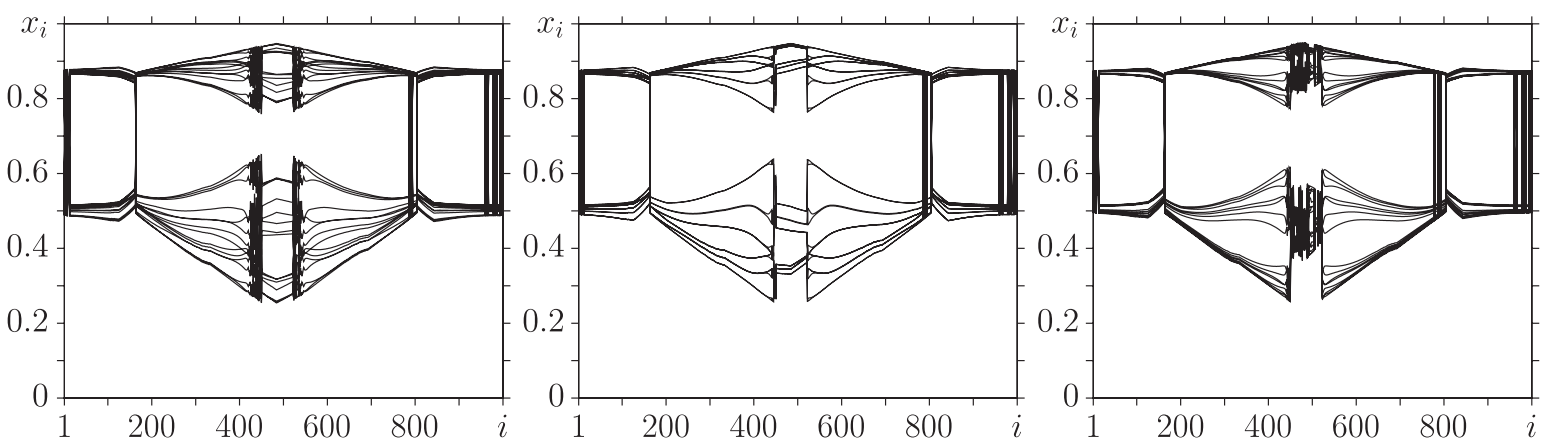

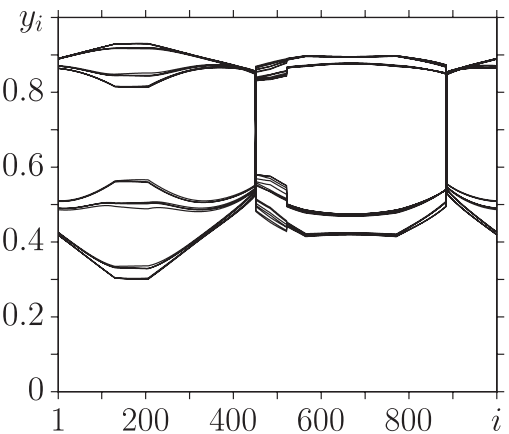

(a)

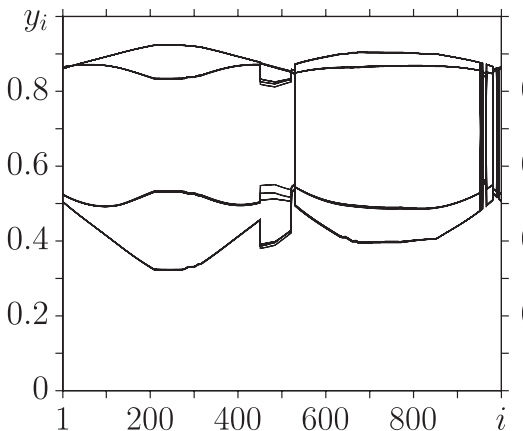

(b)

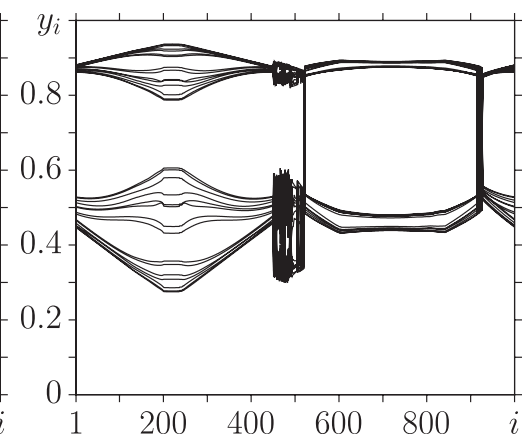

(c)

Fig. 10. Spatio-temporal profiles of the structures in the first (the upper row) and the second (the lower row) layers for various values of interlayer coupling strength $\gamma$ : (a) 0.1 , (b) 0.4 , (c) 0.8 , with the bidirectional coupling via a noncoherent cluster of an amplitude chimera state. The other parameters are as for Fig. 1.

In order to obtain a more detailed analysis of the dynamics of the coupled layers, $\delta_{i}(2.2)$ between the values of $x_{i}$ and $y_{i}$, was calculated for several values of $\gamma$. The results are presented in Fig. 11 for unidirectional (Fig. 11a) and bidirectional (Fig. 11b) interlayer coupling.

It can be seen from the plots that in both cases the greatest similarity in the structures between the two layers takes place precisely in the region of the incoherent cluster of the amplitude chimera (elements in the interval $[450 ; 520]$ ). For these elements, $\delta_{i}$ is always less than 0.1 , and for some values of $\gamma \delta_{i}<0.05$. This fact is quite obvious: it is the elements of interacting

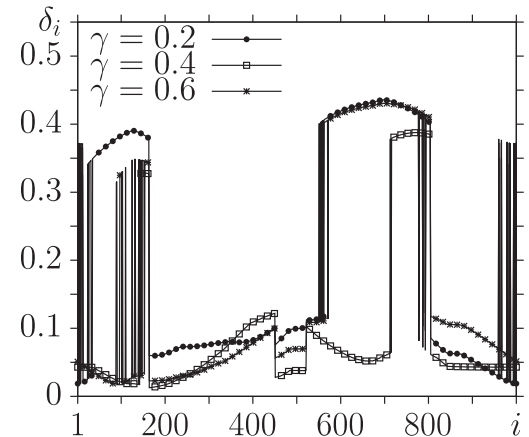

(a)

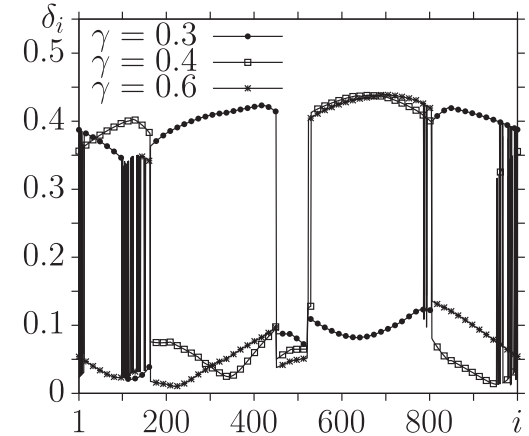

(b)

Fig. 11. Dependences of the first synchronization measure (2.2) for three values of interlayer coupling strength $\gamma$ for unidirectional (a) and bidirectional (b) interlayer coupling in the network (2.1) with the coupling via a noncoherent cluster of an amplitude chimera state. The other parameters are as for Fig. 1. 
layers connected with each other that synchronize first. Subsequently, as the interlayer coupling strength $\gamma$ is varied, their dynamics begins to influence the uncoupled elements to a certain extent, which is due to the presence of the nonlocal intralayer coupling in each layer of the network (2.1). In this case, we can talk about "local" effective cluster synchronization.

2. Coupling via noncoherent clusters in the vicinity of an amplitude chimera. Finally, let us examine the situation where the layers of the network (2.1) are coupled via noncoherent clusters which are located on the left and right of the noncoherent cluster of an amplitude chimera and include oscillators in the intervals [170;450] and $[520 ; 750]$ of the initial structure in the driver layer (Fig. 1a). This case is the most interesting and indicative in terms of observing effective external and mutual synchronization of chimera structures in a network (2.1) in the presence of a cluster interlayer coupling.

As illustrated in the plots of spatio-temporal profiles in Fig. 12, the structure of the driver layer can be replicated well enough in the response layer for unidirectional cluster intercoupling. For bidirectional cluster intercoupling very similar structures are achieved in the layers, as can be seen from Fig. 13.

As can be derived from the dependence $\Delta(\gamma)$ (Figs. 14a, 14b), from the interlayer coupling strength $\gamma=0.1$ and onwards, the synchronization measure (2.3) does not exceed the value 0.1 and fluctuates around $\Delta \approx 0.08$. Moreover, this feature occurs both in the case of unidirectional (Fig. 14a) and bidirectional (Fig. 14b) interlayer coupling. Thus, we can conclude that, for this type of cluster coupling, the phenomena of global effective forced and mutual synchronization are achieved with an accuracy of 0.08 .

The synchronization effects are also observed locally. Figures $14 \mathrm{c}, 14 \mathrm{~d}$, demonstrate the calculation results for $\delta_{i}$ (2.2) in the cases of unidirectional (Fig. 14c) and bidirectional (Fig. 14d) interlayer coupling. As in the previously studied cases, the smallest values of $\delta_{i}$ correspond to the elements in the coupling clusters (intervals $[170 ; 450]$ and $[520 ; 750]$ ), and with the increase of $\gamma \delta_{i}$ tends even to zero for a fairly large number of oscillators in the network. Hence, for this type of cluster interlayer coupling almost all the elements of the coupled layers demonstrate effective synchronization (both forced and mutual) with an accuracy of $\delta_{i} \approx 0.03$. The only exceptions are oscillators belonging to noncoherent clusters of amplitude (interval $[450 ; 520]$ ) and phase (intervals [0; 10], [160; 170], [750; 810], [980; 1000]) chimeras, which remain desynchronized in time with increasing interlayer coupling strength. This is especially noticeable for the elements of the noncoherent clusters of the phase chimera, for which $\delta_{i}>0.37$.

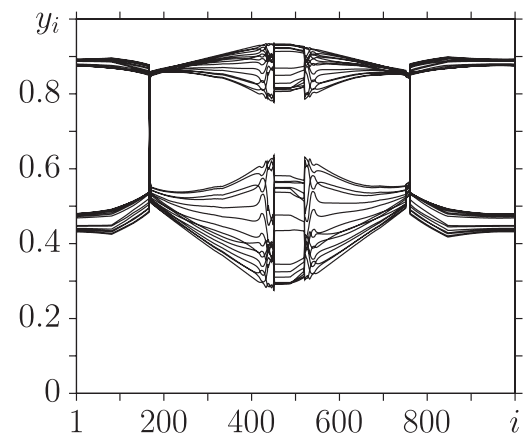

(a)

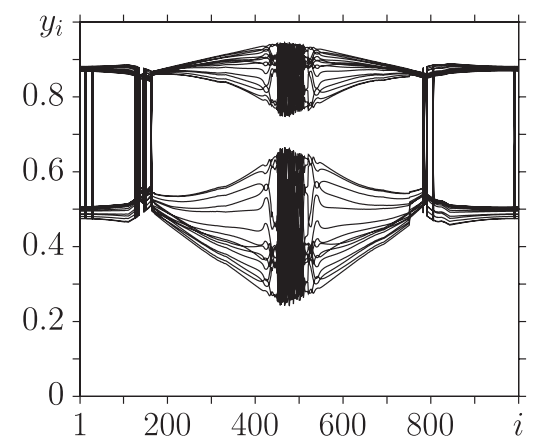

(b)

Fig. 12. Spatio-temporal profiles of the structures in the second $(y)$ layer for two values of interlayer coupling strength $\gamma$ : (a) 0.3, (b) 0.7, with the unidirectional interlayer coupling via the noncoherent clusters. The other parameters are as for Fig. 1. 

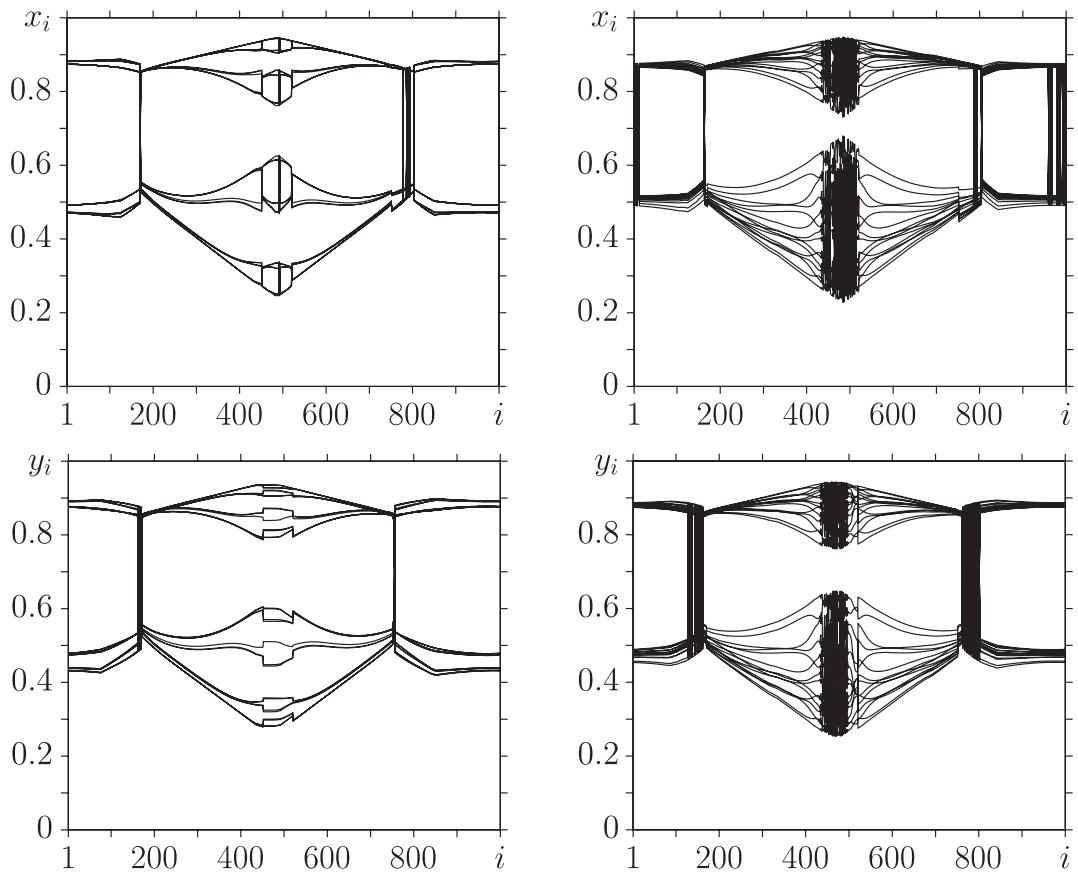

(a)

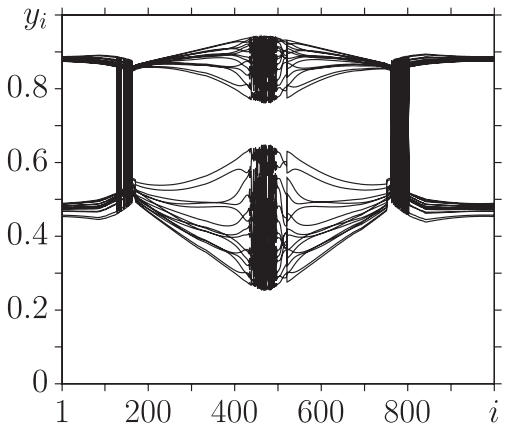

(b)

Fig. 13. Spatio-temporal profiles of the structures in the first (the upper row) and the second (the lower row) layers for various values of interlayer coupling strength $\gamma$ : (a) 0.4 , (b) 0.5 , with the bidirectional coupling via the noncoherent clusters. The other parameters are as for Fig. 1.

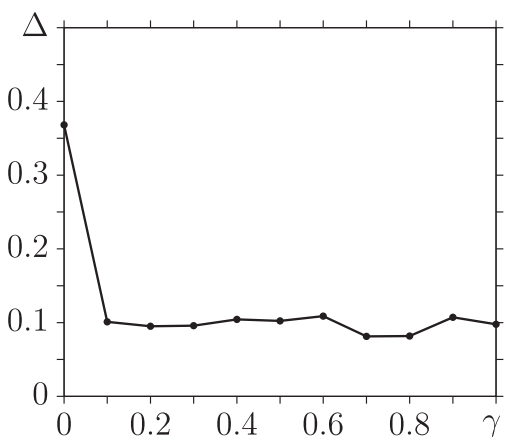

(a)

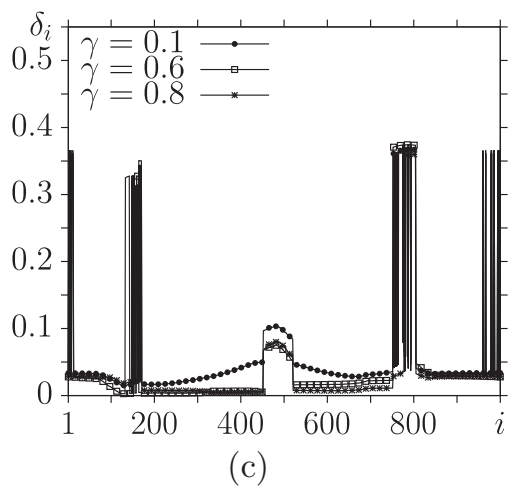

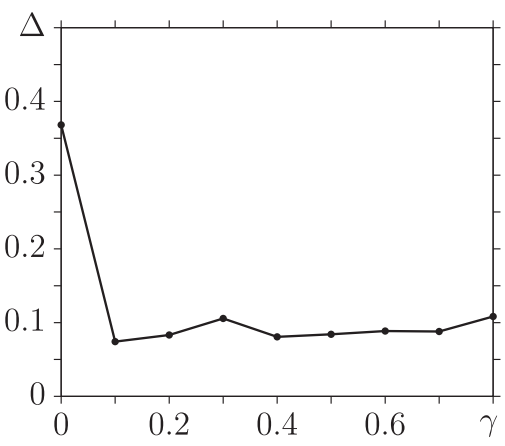

(b)

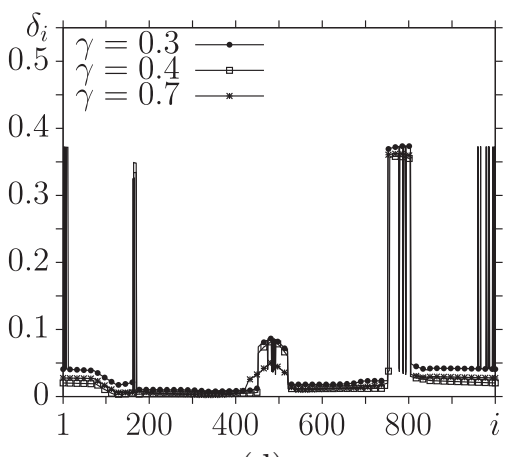

(d)

Fig. 14. Dependences of $\Delta(2.3)$ and $\delta_{i}$ (2.2) on interlayer coupling strength $\gamma$ for unidirectional (a), (c) and bidirectional (b), (d) interlayer coupling via the noncoherent clusters. The other parameters are as for Fig. 1. 


\section{Conclusions}

In this paper, using the example of a two-layer multiplex network of nonlocally coupled identical logistic maps, we have analyzed in detail the effects of forced and mutual synchronization of chimera structures in the presence of inhomogeneity of the interlayer coupling between the layers. When the layers are uncoupled and when randomly distributed initial conditions are assigned, each layer exhibits complex spatio-temporal structures in the form of various chimera structures. We have considered two different configurations for the inhomogeneous interlayer coupling. In the first case, a certain (designated) number of links between the elements were removed randomly. In the second case, the so-called cluster intercoupling was introduced, when the couplings between all the corresponding elements were removed, except for those that correspond to a certain coherent or noncoherent cluster(s) of the first layer structure.

It has been found that in all the cases considered, the sparse interlayer coupling led to a deterioration in the forced and mutual synchronization of chimera states in the two-layer network. Analysis of the influence of the number of removed links on the degree of synchronization effect in the coupled layers with variations in the strength of the intercoupling showed that there is a certain finite region in terms of the number of excluded links and in the strength of the interlayer coupling, in which effective (with a given accuracy) forced and mutual synchronization of chimera states takes place.

In the case where a cluster interlayer coupling is specified, effective (locally) forced and mutual cluster synchronization is observed. Calculations have shown that those regions of chimera structures that correspond to connected clusters are synchronized in the best way. As a result of the studies carried out, clusters of elements were identified, with the introduction of interlayer coupling along which one can observe almost complete forced and mutual synchronization (both locally and globally) of chimera structures. In the case under consideration, these clusters correspond to the regions of incoherence located in the vicinity of a noncoherent cluster of the amplitude chimera.

The results obtained can be useful in modeling the dynamics of real interacting systems in the presence of various types of the connection defects (sparseness) and in assessing their influence on the accuracy of transmission and reproduction of a given state or structure.

\section{References}

[1] Nekorkin, V.I. and Velarde, M. G., Synergetic Phenomena in Active Lattices: Patterns, Waves, Solitons, Chaos, Berlin: Springer, 2002.

[2] Pikovsky, A., Rosenblum, M., and Kurths, J., Synchronization: A Universal Concept in Nonlinear Sciences, Cambridge Nonlinear Sci. Ser., vol. 12, New York: Cambridge Univ. Press, 2001.

[3] Osipov, G., Kurths, J., and Zhou, C., Synchronization in Oscillatory Networks, Berlin: Springer, 2007.

[4] Anishchenko, V.S., Astakhov, V.V., Neiman, A. B., Vadivasova, T.E., and Schimansky-Geier, L., Nonlinear Dynamics of Chaotic and Stochastic Systems: Tutorial and Modern Developments, 2nd ed., Springer Series in Synergetics, Berlin: Springer, 2007.

[5] del Genio, Ch., Gómez-Gardeñes, J., Bonamassa, I., and Boccaletti, S., Synchronization in Networks with Multiple Interaction Layers, Sci. Adv., 2016, vol. 2, no. 11, e1601679, 10 pp.

[6] Boccaletti, S., Pisarchik, A. N., del Genio, Ch. I., and Amann, A., Synchronization: From Coupled Systems to Complex Networks, Cambridge: Cambridge Univ. Press, 2018.

RUSSIAN JOURNAL OF NONLINEAR DYNAMICS, 2021, 17(1), 103-117 
[7] Pecora, L. M., Sorrentino, F., Hagerstrom, A. M., Murphy, Th. E., and Roy, R., Symmetries, Cluster Synchronization, and Isolated Desynchronization in Complex Networks, Nat. Commun., 2014, vol. 5, no. 4079, 8 pp.

[8] Jalan, S. and Singh, A., Cluster Synchronization in Multiplex Networks, Europhys. Lett., 2016, vol. 113, no. 3, 30002, 6 pp.

[9] Andrzejak, R. G., Ruzzene, G., and Malvestio, I., Generalized Synchronization between Chimera States, Chaos, 2017, vol. 27, no. 5, 053114, 6 pp.

[10] Leyva, I., Sendiña-Nadal, I., Sevilla-Escoboza, R., Vera-Avila, V. P., Chholak, P., and Boccaletti, S., Relay Synchronization in Multiplex Networks, Sci. Rep., 2018, vol. 8, Art. No. 1, 12 pp.

[11] Winkler, M., Sawicki, J., Omelchenko, I., Zakharova, A., Anishchenko, V., and Schöll, E., Relay Synchronization in Multiplex Networks of Discrete Maps, Europhys. Lett., 2019, vol. 126, no.5, 50004, 7 pp.

[12] Drauschke, F., Sawicki, J., Berner, R., Omelchenko, I., and Schöll, E., Effect of Topology upon Relay Synchronization in Triplex Neuronal Networks, Chaos, 2020, vol. 30, no. 5, 051104, 7 pp.

[13] Rybalova, E. V., Strelkova, G. I., Schöll, E., and Anishchenko, V.S., Relay and Complete Synchronization in Heterogeneous Multiplex Networks of Chaotic Maps, Chaos, 2020, vol. 30, no. 6, 061104, $10 \mathrm{pp}$.

[14] Shepelev, I. A., Bukh, A. V., Strelkova, G. I., and Anishchenko, V.S., Anti-Phase Relay Synchronization of Wave Structures in a Heterogeneous Multiplex Network of 2D Lattices, Chaos Solitons Fractals, 2021, vol. 143, 110545, 9 pp.

[15] Bukh, A. V., Strelkova, G. I., and Anishchenko, V.S., Synchronization of Chimera States in Coupled Networks of Nonlinear Chaotic Oscillators, Russian J. Nonlinear Dyn., 2018, vol. 14, no.4, pp. 419-433.

[16] Strelkova, G. I., Vadivasova, T.E., and Anishchenko, V.S., Synchronization of Chimera States in a Network of Many Unidirectionally Coupled Layers of Discrete Maps, Regul. Chaotic Dyn., 2018, vol. 23, nos. 7-8, pp. 948-960.

[17] Kasatkin, D. V. and Nekorkin, V.I., Synchronization of Chimera States in a Multiplex System of Phase Oscillators with Adaptive Couplings, Chaos, 2018, vol. 28, no. 9, 093115.

[18] Rybalova, E. V., Vadivasova, T. E., Strelkova, G. I., Anishchenko, V. S., and Zakharova, A. S., Forced Synchronization of a Multilayer Heterogeneous Network of Chaotic Maps in the Chimera State Mode, Chaos, 2019, vol. 29, no. 3, 033134, 9 pp.

[19] Bukh, A. V., Schöll, E., and Anishchenko, V.S., Synchronization of Spiral Wave Patterns in TwoLayer 2D Lattices of Nonlocally Coupled Discrete Oscillators, Chaos, 2019, vol. 29, no. 5, 053105, $7 \mathrm{pp}$.

[20] Bogatenko, T.R., Bukh, A. V., Anishchenko, V.S., and Strelkova, G. I., Synchronization Effects in a Two-Layer Network of Nonlocally Coupled Chaotic Maps with Dissipative and Inertial Intercoupling, Izv. SGU. Novaya Seriya. Fizika, 2020, vol. 20, no. 1, pp. 42-54 (Russian).

[21] Bukh, A. V. and Anishchenko, V.S., Features of the Synchronization of Spiral Wave Structures in Interacting Lattices of Nonlocally Coupled Maps, Russian J. Nonlinear Dyn., 2020, vol. 16, no. 2, pp. 243-257.

[22] Shepelev, I. A., Bukh, A.V., Vadivasova, T.E., and Anishchenko, V.S., Synchronization Effects for Dissipative and Inertial Coupling between Multiplex Lattices, Commun. Nonlinear Sci. Numer. Simul., 2021, vol. 93, 105489, 13 pp.

[23] Gambuzza, L. V., Frasca, M., and Gómez-Gardeñes, J., Intra-Layer Synchronization in Multiplex Networks, Europhys. Lett., 2015, vol.110, no. 2, 20010, 6 pp.

[24] Sevilla-Escoboza, R., Sendiña-Nadal, I., Leyva, I., Gutiérrez, R., Buldú, J. M., and Boccaletti, S., Inter-Layer Synchronization in Multiplex Networks of Identical Layers, Chaos, 2016, vol. 26, no. 6, 065304, 6 pp.

[25] Leyva, I., Sevilla-Escoboza, R., Sendiña-Nadal, I., Gutiérrez, R., Buldú, J. M., and Boccaletti, S., Inter-Layer Synchronization in Non-Identical Multi-Layer Networks, Sci. Rep., 2017, vol.7, Art. No. 45475, 9 pp. 
[26] Vadivasova, T. E., Slepnev, A. V., and Zakharova, A., Control of Inter-Layer Synchronization by Multiplexing Noise, Chaos, 2020, vol. 30, no. 9, 091101, 10 pp.

[27] Leyva, I., Sendiña-Nadal, I., Almendral, J.A., Navas, A., Olmi, S., and Boccaletti, S., Explosive Synchronization in Weighted Complex Networks, Phys. Rev. E, 2013, vol. 88, no. 4, 042808, 7 pp.

[28] Zhang, X., Boccaletti, S., Guan, S., and Liu, Z., Explosive Synchronization in Adaptive and Multilayer Networks, Phys. Rev. Lett., 2015, vol. 114, no. 3, 038701, 5 pp.

[29] Koronovskii, A.A., Kurovskaya, M.K., Moskalenko, O. I., Hramov, A., and Boccaletti, S., SelfSimilarity in Explosive Synchronization of Complex Networks, Phys. Rev. E, 2017, vol.96, no.6, 062312, 6 pp.

[30] Jalan, S., Rathore, V., Kachhvah, A. D., and Yadav, A., Inhibition-Induced Explosive Synchronization in Multiplex Networks, Phys. Rev. E, 2019, vol. 99, no. 6, p. 062305, 10 pp.

[31] Kachhvah, A.D. and Jalan, S., Delay Regulated Explosive Synchronization in Multiplex Networks, New J. Phys., 2019, vol. 21, no. 1, 015006, 11 pp.

[32] Khoshkhou, M. and Montakhab, A., Explosive, Continuous and Frustrated Synchronization Transition in Spiking Hodgkin - Huxley Neural Networks: The Role of Topology and Synaptic Interaction, Phys. D, 2020, vol. 405, no. 10, 132399, 10 pp.

[33] Hong, S. and Chun, Y., Efficiency and Stability in a Model of Wireless Communication Networks, Soc. Choice Welf., 2010, vol. 34, no. 3, pp. 441-454.

[34] Menck, P. J., Heitzig, J., Kurths, J., and Schellnhuber, H. J., How Dead Ends Undermine Power Grid Stability, Nat. Commun., 2014, vol. 5, Art. No. 3969, 8 pp.

[35] Wang, B., Suzuki, H., and Aihara, K., Enhancing Synchronization Stability in a Multi-Area Power Grid, Sci. Rep., 2016, vol. 6, Art. No. 26596, 11 pp.

[36] Khramenkov, V., Dmitrichev, A., and Nekorkin, V., Dynamics and Stability of Two Power Grids with Hub Cluster Topologies, Cybern. Phys., 2019, vol. 8, no. 1, pp. 29-33.

[37] Uhlhaas, P. J. and Singer, W., Neural Synchrony in Brain Disorders: Relevance for Cognitive Dysfunctions and Pathophysiology, Neuron, 2006, vol. 52, no. 1, pp. 155-168.

[38] Chouzouris, T., Omelchenko, I., Zakharova, A., Hlinka, J., Jiruska, P., and Schöll, E., Chimera States in Brain Networks: Empirical Neural vs. Modular Fractal Connectivity, Chaos, 2018, vol. 28, no. 4, 045112, $10 \mathrm{pp}$.

[39] Ramlow, L., Sawicki, J., Zakharova, A., Hlinka, J., Claussen, J. C., and Schöll, E., Partial Synchronization in Empirical Brain Networks As a Model for Unihemispheric Sleep, Europhys. Lett., 2019, vol. 126 , no. $5,50007,7$ pp.

[40] Aguirre, J., Sevilla-Escoboza, R., Gutiérrez, R., Papo, D., and Buldú, J. M., Synchronization of Interconnected Networks: The Role of Connector Nodes, Phys. Rev. Lett., 2014, vol. 112, no. 24, 248701, $5 \mathrm{pp}$.

[41] Leyva, I., Sendiña-Nadal, I., Almendral, J. A., and Sanjuán, M. A., Sparse Repulsive Coupling Enhances Synchronization in Complex Networks, Phys. Rev. E, 2006, vol. 74, no. 5, 056112, 7 pp.

[42] Rybalova, E.V., Strelkova, G.I., Anishchenko, V.S., Impact of Sparse Inter-Layer Coupling on the Dynamics of a Heterogeneous Multilayer Network of Chaotic Maps, Chaos Solitons Fractals, 2021, vol. 142, Art. 110477.

[43] Omelchenko, I., Maistrenko, Yu., Hövel, Ph., and Schöll, E., Loss of Coherence in Dynamical Networks: Spatial Chaos and Chimera States, Phys. Rev. Lett., 2011, vol. 106, no. 23, 234102, 4 pp.

[44] Bogomolov, S., Slepnev, A., Strelkova, G., Schöll, E., and Anishchenko, V., Mechanisms of Appearance of Amplitude and Phase Chimera States in Ensembles of Nonlocally Coupled Chaotic Systems, Commun. Nonlinear Sci. Numer. Simul., 2017, vol.43, pp. 25-36. 